\title{
Poling-assisted bleaching of metal-doped nanocomposite glass
}

\author{
Olivier Deparis ${ }^{\text {a) }}$ and Peter G. Kazansky \\ Optoelectronics Research Centre, University of Southampton, SO17 1BJ, United Kingdom
}

Amin Abdolvand, Alexander Podlipensky, Gerhard Seifert, and Heinrich Graener

Physics Department, Martin-Luther-University Halle-Wittenberg, D-06099 Halle, Germany

(Received 12 April 2004; accepted 11 June 2004)

\begin{abstract}
Thermal poling of soda-lime glass which was doped with spherical or ellipsoidal silver nanoparticles has revealed what we believe to be a phenomenon of general interest in the physics of nanocomposite materials: The field-assisted dissolution of metal nanoparticles embedded in glass. Macroscopically, this phenomenon manifested itself as poling-assisted bleaching of the glass in the sense that the glass became more (or even completely) transparent under the anode. The phenomenon is physically interpreted in terms of the ionization of metal nanoclusters followed by the removal of ions from the clusters and their drift in the depth, under the action of the extremely high electric field which is created underneath the anodic surface during poling. The underlying physical mechanism is expected to offer unique opportunities for the control of structural and optical properties of nanocomposite glasses. (C) 2004 American Institute of Physics.
\end{abstract}

[DOI: $10.1063 / 1.1779966]$

Poling techniques have been intensively exploited for the purpose of inducing second-order optical nonlinearity in various types of glass. ${ }^{1,2}$ Among the available techniques, thermal poling has become very popular thanks to its simplicity and reliability. The underlying physical principle that is generally accepted is the following: The inversion symmetry of the glass matrix is broken by the internal electrostatic field which is frozen in the glass after poling and, as a result, second-order nonlinear response to intense light excitation is allowed. Enhancement of the second-order nonlinearity in poled glass became a concern as soon as it was realized that the induced second-order nonlinear susceptibility, $\chi^{(2)}$, is limited by the intrinsic third-order nonlinear susceptibility, $\chi^{(3)}$, and by the dielectric breakdown strength of the glass, according to the $\chi^{(2)}=3 E_{\mathrm{dc}} \chi^{(3)}$ model. ${ }^{3}$ Recently, large second-harmonic signal was observed in silica waveguides poled with silver-containing electrode, which was interpreted in terms of resonance enhancement of $\chi^{(2)}$ in the vicinity of the surface plasmon resonance (SPR) frequency of metal nanoparticles embedded in glass. ${ }^{4}$ Injection of silver ions from the electrode was believed to lead to aggregation of silver metal nanoclusters which were responsible for SPR enhancement of $\chi^{(2)}{ }^{4}$ On the other hand, without treatment such as poling, Podlipensky and co-workers ${ }^{5}$ observed recently resonant $\chi^{(2)}$ in thin layers of ellipsoidal silver nanoparticles embedded in silica glass. In the present context, silica glass doped with silver nanoparticles appears to be an interesting basis for further studies. We have carried out thermal poling experiments on such a glass with the aim of investigating possible SPR enhancement of the poling-induced second-order nonlinearity. Unexpectedly, our experiments revealed a phenomenon which is believed to be of general interest in the physics of nanocomposite materials: The fieldassisted dissolution of metal nanoparticles embedded in

\footnotetext{
a)Permanent address: Faculté Polytechnique de Mons, Electromagnetism and Telecommunications Department, B-7000 Mons, Belgium; electronic mail: deparis@telecom.fpms.ac.be
}

glass. Results related to this phenomenon are reported in this letter together with discussions on its physical origin.

Soda-lime float glass was used as the substrate for $\mathrm{Ag}^{+}-\mathrm{Na}^{+}$ion exchange $\left(72.5 \mathrm{SiO}_{2}, 14.4 \mathrm{Na}_{2} \mathrm{O}, 0.7 \mathrm{~K}_{2} \mathrm{O}, 6.1\right.$ $\mathrm{CaO}, 4.0 \mathrm{MgO}, 1.5 \mathrm{Al}_{2} \mathrm{O}_{3}, 0.1 \mathrm{Fe}_{2} \mathrm{O}_{3}, 0.1 \mathrm{MnO}, 0.4 \mathrm{SO}_{3}$ in wt \%). Thermal annealing of ion-exchanged samples in a $\mathrm{H}_{2}$ reduction atmosphere resulted in the formation of spherical silver nanoparticles of $30-40 \mathrm{~nm}$ mean diameter in a nearsurface layer of approximately $6 \mu \mathrm{m}$ thickness. The metal filling volume factor decreased in the depth from $\approx 0.8$ to 0.001 . This gradient allowed us to produce samples with a very low filling factor by removing the upper part of the nanoparticle layer (e.g., sample No. 9). Tensile deformation and simultaneous heating of $1 \mathrm{~mm}$ thick samples with spherical nanoparticles produced $0.2 \mathrm{~mm}$ thick samples with elongated (ellipsoidal) nanoparticles which were uniformly oriented in a near-surface layer of approximately $1 \mu \mathrm{m}$ thickness. Characteristics of the samples are summarized in Table I. For comparison purposes, one sample with spherical nanoparticles (No. 4bis) was polished down to the same thickness as samples with ellipsoidal nanoparticles.

TABLE I. Samples under test: $\mathrm{Ag}^{+}-\mathrm{Na}^{+}$ion exchanged soda-lime glass (substrate), soda-lime glass containing Ag nanoparticles of ellipsoidal shape (nanocomposite $E$ ), or spherical shape (nanocomposite $S$ ). $L$ : Sample thickness, and $d$ : Thickness of the near-surface layer in which $\mathrm{Ag}$ nanoparticles are embedded. Color of the pristine glass and amount of poling-assisted bleaching of the glass are indicated. All samples (except No. 3) were poled with the nanoparticle layer facing the anode (cathode). All samples (except No. 8) were poled at $1 \mathrm{kV}$ and $280{ }^{\circ} \mathrm{C}\left(4 \mathrm{kV}\right.$ and $\left.25^{\circ} \mathrm{C}\right)$.

\begin{tabular}{cccccc}
\hline $\begin{array}{c}\text { Sample } \\
\text { No. }\end{array}$ & Type of material & $\begin{array}{c}L \\
(\mathrm{~mm})\end{array}$ & $\begin{array}{c}d \\
(\mu \mathrm{m})\end{array}$ & Color & $\begin{array}{c}\text { Poling-assisted } \\
\text { bleaching }\end{array}$ \\
\hline 10 & Substrate & 1.0 & - & Transparent & $\ldots$ \\
1 & Nanocomposite $E$ & 0.2 & $\sim 1.0$ & Dark green & Total \\
3 & Nanocomposite $E$ & 0.2 & $\sim 1.0$ & Dark grey & Absent \\
4 & Nanocomposite $S$ & 1.0 & $\sim 6.0$ & Dark orange & Partial \\
4 bis & Nanocomposite $S$ & 0.2 & $\sim 6.0$ & Dark orange & Partial \\
9 & Nanocomposite $S$ & 1.0 & $<1.0$ & Faint yellow & Absent \\
8 & Nanocomposite $S$ & 1.0 & $\sim 6.0$ & Orange & Absent \\
\hline \hline
\end{tabular}



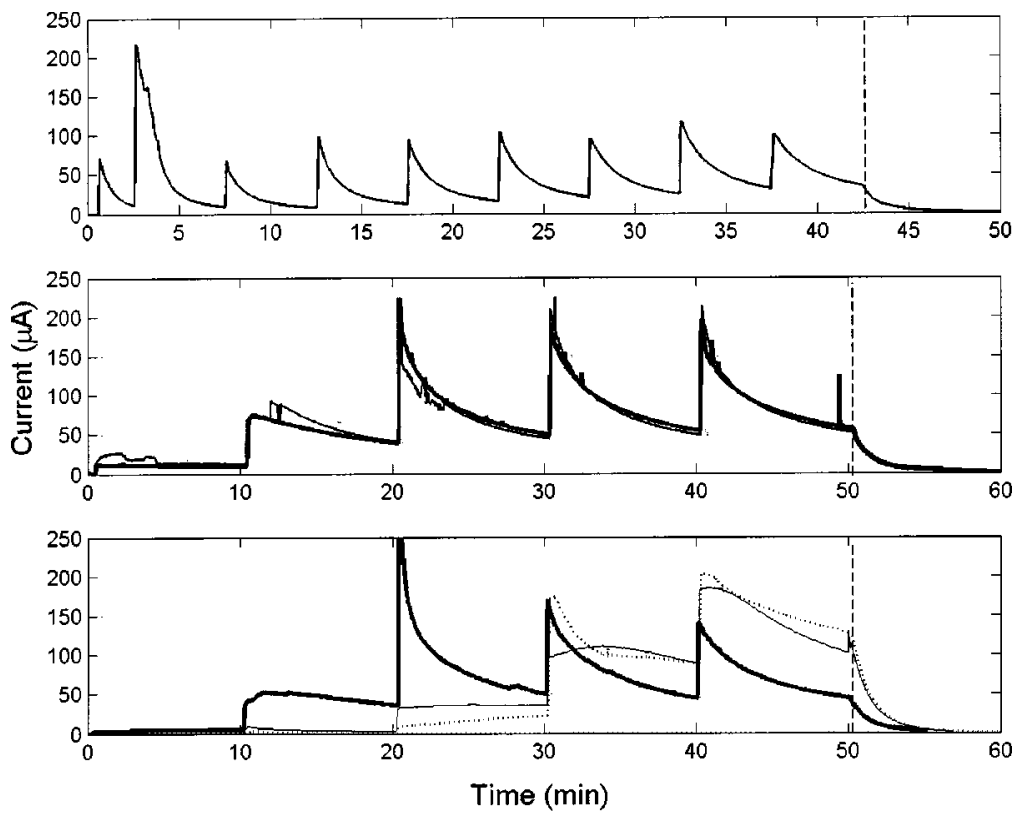

FIG. 1. Evolution of the current flowing through the sample as a function of time during thermal poling at $280^{\circ} \mathrm{C}$. Top chart: Ion-exchanged sample No. 10; middle chart: Samples containing ellipsoidal Ag nanoparticles (No. 1: Thick line, No. 3: Thin line); bottom chart: samples containing spherical Ag nanoparticles (No. 4: Thin line, No. 4bis: Thick line, No. 9: Dotted line). The voltage was applied step by step up to a final value of $1.0 \mathrm{kV}$ (top chart No. 1: First step of $0.2 \mathrm{kV}$ during $2 \mathrm{~min}$, then steps of $0.1 \mathrm{kV}, 5 \mathrm{~min}$ each; middle and bottom charts: steps of $0.2 \mathrm{kV}, 10 \mathrm{~min}$ each). Vertical dashed lines indicate the beginning of cooling of the sample with the voltage still applied.
Thermal poling was carried out at $280^{\circ} \mathrm{C}$ and $1 \mathrm{kV}$ in an air atmosphere inside an oven using pressed-contact steel electrodes $(6 \times 9 \mathrm{~mm})$. Because soda-lime glasses possess high ionic conductivity, special procedures have to be employed for poling. ${ }^{6}$ The procedure we used to pole our samples consisted of applying the voltage in small amplitude steps in such a way that, during each step, the current never exceeded a few hundreds of microamperes. The idea behind this procedure is to let a high-resistivity layer progressively form under the anodic surface as a result of ion migration. This procedure was tested on a $1 \mathrm{~mm}$ thick ion-exchanged soda-lime sample (No. 10). The ion-exchanged sample exhibited typical behavior of soda-lime glass during poling: At each step, the current decreased with time (peak current remained below $\sim 200 \mu \mathrm{A}$ ) and the decay of the current became slower from step to step, indicating that the resistivity of the ion-depleted region increased progressively (Fig. 1-top chart). A similar procedure was then used to pole samples doped with silver nanoparticles. For all samples (except No. 3), the electrode polarity was chosen in such a way that the silver-doped layer faced the anode. Samples doped with silver nanoparticles exhibited substantially different poling current dynamics in comparison with the ionexchanged sample (Fig. 1). In these samples, the nearsurface layer is depleted in sodium ions and contains isolated clusters of metallic silver. Therefore, the ionic conductivity in that layer is expected to be very low, a feature that is consistent with the much smaller current which was observed during the first two or three steps of the poling. Afterward, the conductivity increased dramatically (the evolution of the current with time became similar to that in the ion-exchanged sample). This sudden change of behavior is believed to be related with the onset of the dissolution of silver nanoparticles, as will be discussed hereafter.

Examination of the samples after poling has revealed amazing effects. After poling of a sample doped with ellipsoidal particles (No. 1), the glass region under the positive electrode had become completely transparent with sharp edges corresponding perfectly to the electrode size (Fig. 2). Since the original color of the glass (here dark green) was due to the presence of silver particles, the bleaching of the glass in the poled region is obviously due to the dissolution of these particles. From a macroscopic point of view, we call this effect "poling-assisted bleaching" (PAB). The sample was cut in the middle of the poled region for further investigations. Scanning electron microscope (SEM) images of the cross section of one-half of the sample showed that there was no more silver nonoparticles (SEM results will be published elsewhere). Thermal annealing treatment of the other one-half of the sample led to reaggregation of silver nanoparticles, as was indicated by the partial recovery of the original glass color in the poled region. The latter result gave us evidence that silver was still present in the glass after poling but in the form of ions rather than metal. We believe that the macroscopic PAB effect is the manifestation of a microscopic physical phenomenon which involves successively the ionization of metal nanoclusters, the removal of ions from the clusters and the drift of the ions in the depth, all of these three processes being driven by the extremely high electric field which is created underneath the anodic surface during poling. Another sample doped with ellipsoidal particles (No. 3) was poled with the silver-doped layer facing

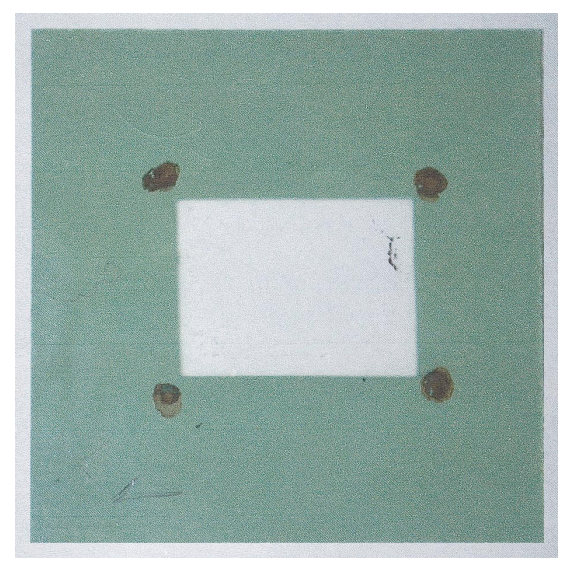

FIG. 2. (Color) Photograph image of samples No. 1 after poling. The glass became transparent under the anode after poling (i.e., PAB occurred). The sharp edges of the bleached region correspond perfectly to the electrode size $(6 \times 9 \mathrm{~mm})$. 
the cathode instead of the anode. After poling, no change of color was observed at all in the poled area. In spite of different amounts of silver in sample Nos. 3 and 1, these experiments confirmed that the bleaching was related to polingassisted modifications of the glass region under the positive electrode. The PAB effect was also observed after poling of samples doped with spherical particles (Nos. 4 and 4bis). Only partial bleaching of the glass under the poled area was observed (the original dark orange color became yellowish) but the sharp edge feature was preserved. To which extent the sample was bleached did not qualitatively depend on its thickness $(1.0 \mathrm{~mm}$ or $0.2 \mathrm{~mm})$. On the other hand, no change of color was observed after poling of a $1 \mathrm{~mm}$ thick sample which was doped with spherical particles but in a much reduced amount (No. 9). At room temperature, high voltage $(4 \mathrm{kV})$ was applied to a $1 \mathrm{~mm}$ thick sample doped with spherical particles (No. 8). No change of color was observed after that treatment for $5 \mathrm{~min}$ and $15 \mathrm{~min}$. The latter result showed us that thermal poling (i.e., the combined action of high voltage and high temperature) was definitively at the origin of the bleaching and justified our denomination (PAB).

The bleaching we have observed in our samples takes place in the presence of an intense dc electric field at an elevated temperature. The physical origin of the polingassisted bleaching of the glass is believed to be the fieldassisted dissolution of embedded metal nanoparticles. At room temperature, the electric field is constant across the sample (no charge migration can take place) and typical field strength $\left(4 \times 10^{6} \mathrm{~V} / \mathrm{m}\right.$ in the experiment on sample No. 8$)$ is not high enough to cause dissolution of the silver nanoparticles. Thermal poling, on the other hand, creates conditions by which much higher field strength is achieved in a thin layer underneath the anodic surface of the sample. In the case of the ion-exchanged sample, a high-resistivity iondepleted layer is formed under the anodic surface as positive ion migration proceeds and, like in a voltage divider, the voltage tends to drop across the depletion layer instead of the whole bulk. This picture remains valid, in general, in the case of the samples doped with silver nanoparticles but one must take into account that the metal-glass nanocomposite layer, where conductivity is different from the substrate, modifies both the dynamics of formation of the depletion region and the distribution of the internal electric field, as is suggested by the evolution of the current during the first steps of poling (Fig. 1). The order of magnitude of the electric-field strength in the region underneath the anodic surface $\left(E_{\mathrm{dc}}\right)$ can be roughly estimated as the externally applied voltage divided by the thickness of the depletion region. In our experiments, $E_{\mathrm{dc}}$ is at least higher than 2 $\times 10^{8} \mathrm{~V} / \mathrm{m}$ if we assume that a typical thickness of the depletion layer is equal to $1 \mu \mathrm{m}$ and if we take $V=0.2 \mathrm{kV}$ (i.e., the amplitude of the first voltage step). The actual electric-field strength can be even higher due to local field enhancement around metal clusters. ${ }^{7}$ The metal volume filling factor is therefore an important parameter from this point of view. In sample No. 9 (very low filling factor), we think that field-assisted dissolution of nanoparticles did not take place because the strength of the electric field surrounding the particles was not high enough due to a longer average distance between particles. The overlap between the depletion layer and the metal-doped layer is critical in order to observe the bleaching. In our samples, the typical thickness of the metal-doped layer and its location near the surface ensured that overlap was reasonably good when the metaldoped layer faced the anode. As a matter of fact no overlap could be achieved when the metal-doped layer faced the cathode. The fact that the field strength under anodic surface does not depend on sample thickness (in a very reasonable approximation) is consistent with our observation of similar amounts of bleaching in thin $(0.2 \mathrm{~mm})$ and thick $(1.0 \mathrm{~mm})$ samples of identical composition (Nos. 4bis and 4, respectively). The setup we used for poling did not allow us to detect (even qualitatively by eyes) the onset of the bleaching during poling. Therefore, we were not able to determine during which voltage step the bleaching appeared and how much time it took to complete. Monitoring of the sample aspect during poling at various voltages would be extremely useful in order to determine threshold and dynamics of the physical phenomenon.

In summary, we reported experimental evidences of field-assisted dissolution of metal nanoparticles embedded in glass. This phenomenon is believed to be of general interest in the physics of nanocomposite materials. Beyond the already interesting capability to make the glass totally transparent under micrometric areas with sharp edges, the underlying physical mechanism is potentially promising for the control of structural and optical properties of nanocomposite materials, such as metal-doped or semiconductor-doped nanocomposite glasses.

The authors are very grateful to CODIXX AG in Barleben (Germany) for providing the samples for this study. One of the authors (O.D.) acknowledges the Interuniversity Attraction Pole IAP V/18 programme of the Belgian Science Policy.

${ }^{1}$ R. A. Myers, N. Mukherjee, and S. R. J. Brueck, Opt. Lett. 16, 1732 (1991).

${ }^{2}$ P. G. Kazansky, A. Kamal, and P. St. J. Russell, Opt. Lett. 18, 693 (1993). ${ }^{3}$ P. G. Kazansky and P. St. J. Russell, Opt. Commun. 110, 611 (1994).

${ }^{4}$ J. Arentoft, M. Kristensen, K. Pedersen, S. I. Bozhevolnyi, and P. Shi, Electron. Lett. 36, 1635 (2000).

${ }^{5}$ A. Podlipensky, J. Lange, G. Seifert, H. Graener, and I. Cravetchi, Opt. Lett. 28, 716 (2003).

${ }^{6}$ F. C. Garcia, I. C. S. Carvalho, E. Hering, W. Margulis, and B. Lesche, Appl. Phys. Lett. 72, 3252 (1998).

${ }^{7}$ K. L. Kelly, E. Coronado, L. L. Zhao, and G. C. Schatz, J. Phys. Chem. B 107, 668 (2003). 